\title{
Conhecimento da equipe de enfermagem sobre inserção, manutenção e complicações relacionados ao cateter venoso periférico
}

\author{
Knowledge of the nursing team on insertion, maintenance and complications related to the \\ peripheral venous catheter
}

Conocimiento del equipo de enfermería sobre inserción, mantenimiento y complicaciones relacionadas con el catéter venoso periférico

Bruna Katerine Godinho Gomes ${ }^{1 *}$, Aurelina Gomes e Martins ${ }^{1}$, Joanilva Ribeiro Lopes ${ }^{1}$, Henrique Andrade Barbosa ${ }^{1}$, Daniella Fagundes Souto ${ }^{1}$, Ana Paula Ferreira Maciel ${ }^{1}$, Bruna Emanuelle Santos ${ }^{1}$, Vinícius Gomes e Martins² ${ }^{2}$ Daniela Oliveira Lima Magalhães ${ }^{1}$.

\section{RESUMO}

Objetivo: Avaliar o conhecimento da equipe de enfermagem sobre a inserção, manutenção e complicações relacionadas ao uso do cateter venoso periférico. Métodos: Pesquisa transversal, descritiva, exploratória e quantitativa, desenvolvida em um Hospital Escola do município de Montes Claros- MG. Amostra composta por 189 sujeitos selecionados de forma aleatória. Aplicou-se dois questionários, um sociodemográfico e funcional, e outro com questões baseadas no "Instrumento de Registro de Busca Ativa", além das recomendações da Agência Nacional de Vigilância Sanitária. Realizou-se pré-teste com os profissionais do setor de pediatria do referido serviço. Resultados: Após aplicação do pré-teste percebeu-se a necessidade de alteração apenas no layout dos instrumentos. Participaram deste estudo 189 profissionais. A maioria eram mulheres $(63,5 \%)$, técnicas em enfermagem $(83,6 \%)$. Sobre o material adequado para fixação dos cateteres, $(73,0 \%)$ relataram que é o esparadrapo e/ou fita adesiva microporosa. Referindo-se à flebite $(49,7 \%) \mathrm{e}$ infiltração e extravasamento $(43,4 \%)$ das profissionais demarcaram sinais/sintomas que caracterizam adequadamente estas complicações. Conclusão: Observou-se significância estatística entre profissão e conhecimento sobre as complicações flebite, infiltração e extravasamento e sobre o material ideal para fixação dos cateteres periféricos.

Palavras-chave: Cateterismo periférico, Flebite, Equipe de enfermagem.

\begin{abstract}
Objective: To evaluate the knowledge of the nursing team about the insertion, maintenance and complications related to the use of the peripheral venous catheter. Methods: Cross-sectional, descriptive, exploratory and quantitative research, developed at a Teaching Hospital in the municipality of Montes Claros- MG. Sample composed of 189 subjects selected at random. Two questionnaires were applied, one sociodemographic and functional, and the other with questions based on the "Active Search Registration Instrument", in addition to the recommendations of the National Health Surveillance Agency. A pre-test was carried out with the professionals in the pediatric sector of that service. Results: After applying the pre-test, it was noticed the need to change only the layout of the instruments. 189 professionals participated in this study. Most were women $(63.5 \%)$, nursing technicians $(83.6 \%)$. About the appropriate material for fixing the catheters, $(73.0 \%)$ reported that it is the adhesive tape and / or microporous adhesive tape. Referring to phlebitis (49.7\%) and
\end{abstract}

1 Universidade Estadual de Montes Claros (UNIMONTES), Montes Claros - MG.

*E-mail: brunnahk@gmail.com

${ }^{2}$ Faculdades Integradas Pitágoras (FIPMoc), Montes Claros - MG.

SUBMETIDO EM: 4/2020

ACEITO EM: 5/2020

PUBLICADO EM: 5/2020

REAS/EJCH | Vol.12(8) | e3408 | DOI: https://doi.org/10.25248/reas.e3408.2020 Página 1 de 10 
infiltration and leakage (43.4\%) of the professionals demarcated signs / symptoms that adequately characterize these complications. Conclusion: There was statistical significance between profession and knowledge about phlebitis, infiltration and leakage complications and the ideal material for fixing peripheral catheters.

Keywords: Peripheral catheterization, Phlebitis, Nursing staff.

\section{RESUMEN}

Objetivo: Evaluar el conocimiento del equipo de enfermería sobre la inserción, el mantenimiento y las complicaciones relacionadas con el uso del catéter venoso periférico. Métodos: Investigación transversal, descriptiva, exploratoria y cuantitativa, desarrollada en un Hospital Docente del municipio de Montes ClarosMG. Muestra compuesta por 189 sujetos seleccionados al azar. Se aplicaron dos cuestionarios, uno sociodemográfico y funcional, y el otro con preguntas basadas en el "Instrumento de registro de búsqueda activa", además de las recomendaciones de la Agencia Nacional de Vigilancia Sanitaria. Se realizó una prueba previa con los profesionales del sector pediátrico de ese servicio. Resultados: Después de aplicar la prueba previa, se notó la necesidad de cambiar solo el diseño de los instrumentos. 189 profesionales participaron en este estudio. La mayoría eran mujeres $(63.5 \%)$, técnicos de enfermería (83.6\%). Sobre el material apropiado para la fijación de los catéteres, (73.0\%) informó que es la cinta adhesiva y / o cinta adhesiva microporosa. Refiriéndose a flebitis (49.7\%) e infiltración y fuga (43.4\%) de los profesionales delimitaron signos / síntomas que caracterizan adecuadamente estas complicaciones. Conclusión: Hubo significación estadística entre la profesión y el conocimiento sobre las complicaciones de flebitis, infiltración y fuga y el material ideal para la fijación de catéteres periféricos.

Palabras clave: Cateterismo periférico, Flebitis, Equipo de enfermería.

\section{INTRODUÇÃO}

O Acesso Venoso Periférico (AVP) consiste na colocação de um dispositivo intravenoso, conhecido como cateter, em uma veia periférica para a infusão de drogas e fluídos. Trata-se de um procedimento invasivo realizado por profissionais da enfermagem, sendo muito utilizado na assistência à saúde em pacientes com terapia intravenosa (TI) (BARBOSA M, et al., 2015).

Os cateteres utilizados para a punção venosa são produtos de reprocessamento proibido por esta razão, em casos de insucesso no procedimento, os mesmos não podem ser usados para uma nova tentativa de punção (AGÊNCIA NACIONAL DE VIGILÂNCIA SANITÁRIA, 2017).

Conforme a Agência Nacional de Vigilância Sanitária (ANVISA), as veias de escolha para o AVP em adultos são as das superfícies dorsal e ventral dos antebraços evitando-se a inserção de cateter em membro comprometido por lesões com veias danificadas e regiões de articulações além do membro dominante ou inferior, neste, devido maior risco de complicações (AGÊNCIA NACIONAL DE VIGILÂNCIA SANITÁRIA, 2013).

Os eventos adversos relacionados ao uso de cateter periférico são atribuídos a uma série de fatores entre eles o tipo de dispositivo selecionado, o preparo do local de inserção, a técnica, o tipo de infusão, o curativo e o tempo de permanência podem ser responsáveis por infiltrações, extravasamentos e flebite. Além do mais, o sítio de inserção do cateter é considerado uma porta de entrada para microrganismos presentes na pele que podem alcançar o fluxo intravascular (DANSKI MTR, et al., 2016b).

Para uma punção de qualidade e manutenção adequada do AVP é preciso que os profissionais de saúde possuam capacitação técnico-científica, boas medidas de higiene, materiais adequados, de forma a garantir uma assistência segura aos pacientes. Os eventos adversos são processos infecciosos que podem prolongar o tempo de internação nos serviços de saúde, além de elevar consideravelmente os custos com o cuidado (BITENCOURT et al., 2018; SANTOS DS et al., 2016). 
Flebite é a inflamação da parede da veia causada por traumatismos, substâncias químicas irritantes ou contaminação bacteriana, sempre desencadeia sinais de inflamação aguda podendo ou não ocorrer febre baixa. Se não tratada corretamente, pode evoluir para tromboflebite, facilmente notado na palpação como um cordão fibroso por baixo da pele que pode migrar para o pulmão, e provocar até mesmo uma embolia pulmonar (ANVISA, 2013; OLIVEIRA ECS, et al., 2016; ENES SMS, et al., 2016; TERTULIANO AC, et al., 2014; DANSKI MTR, et al., 2016a).

Infiltração consiste no deslocamento do cateter que causa a administração de fármacos não vesicantes no espaço extravascular. Já o extravasamento, é a infiltração de soluções vesicantes para fora da rede venosa. Os sinais indicativos são: rubor, calor, dor e edema, podendo ou não haver formação de bolhas e até mesmo ulceração (INFUSION NURSES SOCIETY, 2016; BRAGA LM, et al., 2018).

Algumas destas complicações são passíveis de prevenção pela equipe de enfermagem. Cuidados com o curativo e atenção na identificação correta do cateter para controle de seu tempo de permanência facilita a avaliação diária e reduz a ocorrência de eventos adversos (MURASSAKI ACY, et al., 2013)

Para que a prática de enfermagem seja adequada é necessário conhecer tais eventos do AVP, a fim de evitá-las. O conhecimento técnico-científico dos enfermeiros e sua equipe sobre a TI garantem o êxito no tratamento, qualidade do cuidado prestado, possibilitando redução das complicações, tornando-se imprescindível o conhecimento das melhores práticas de cuidado cientificamente comprovadas (DANSKI MTR, et al.,2016a).

Taxas de incidências de complicações vêm sendo utilizadas como um indicador de qualidade da assistência de enfermagem (OLIVEIRA ECS, et al.,2016; SILVA ACA, et al.,2016). No entanto percebe-se ainda a subnotificação de flebite e infiltração (BARBOSA MH, et al., 2016; FEREZIN TPM, et al., 2017). Pesquisadores apontam que a intensa demanda de trabalho muitas vezes impede a detecção e notificação de complicações (SILVA ACA, et al.,2016).

Em síntese, na TI, os cuidados de enfermagem estão voltados para a manutenção de um acesso venoso seguro, com o menor risco para desenvolvimento de infecções locais ou sistêmicas. Não obstante, faz-se necessário conhecimento sobre a técnica correta de inserção, dos materiais que podem ser utilizados, do tempo adequado de permanência do cateter, da necessidade da realização de anotações sobre a evolução e de notificações de complicações. Dessa forma a equipe de enfermagem assume um papel de destaque na terapia venosa periférica ao agregar as habilidades necessárias para fornecer um cuidado seguro e de qualidade (BARBOSA M, et al., 2015; TERTULIANO AC, et al., 2014).

Diante do exposto questionou-se: "Qual o conhecimento da equipe de enfermagem acerca da inserção, manutenção e complicações relacionadas ao uso de cateter venoso periférico?". O objetivo deste estudo foi avaliar o conhecimento da equipe de enfermagem sobre a inserção, manutenção e complicações relacionados ao uso cateter venoso periférico.

\section{MÉTODOS}

Esta é uma pesquisa transversal, de natureza descritiva, exploratória com abordagem quantitativa que possibilitou avaliar o conhecimento da equipe de enfermagem sobre as complicações relacionadas à inserção e manutenção do cateter venoso periférico (CVP).

A pesquisa foi desenvolvida com profissionais de enfermagem alocados na clínica médica (CM), clínica cirúrgica (CC), bloco cirúrgico (BC), bloco obstétrico (BO), maternidade (MAT), pronto socorro (PS) e unidade de terapia intensiva adulto (UTI) de um hospital escola no município de Montes Claros - Minas Gerais.

Este serviço contava com 251 profissionais, sendo 55 enfermeiros e 196 técnicos em enfermagem. Para cálculo do tamanho da amostra observou-se um nível de significância de 95\% e erro amostral de 5\%, foi acrescido $22,5 \%$ de margem de segurança, totalizando 189 profissionais de enfermagem, sendo 31 Enfermeiros e 158 Técnicos em Enfermagem. Foi realizado cálculo para garantir a mesma representatividade de profissionais de cada um dos setores hospitalares e estes foram selecionados de forma aleatória. 
Para composição da amostra foram incluídos os profissionais que se encontravam em pleno exercício profissional por pelo menos três meses do período estabelecido para a coleta de dados, sendo excluídos os profissionais alocados nos outros setores hospitalares.

Com o objetivo de nortear a coleta de dados foram aplicados dois instrumentos, o primeiro, um questionário com dados sociodemográficos e funcionais. O segundo, um questionário baseado no "Instrumento de Registro de Busca Ativa", elaborado e validado por Vituri em 2009 (VITURI DW, MATSUDA LM, 2009), e as recomendações da Anvisa, caderno 4, Medidas de Prevenção de Infecção Relacionada à Assistência à Saúde de 2013 (AGÊNCIA NACIONAL DE VIGILÂNCIA SANITÁRIA, 2013). Essa pesquisa foi aprovada pela Coordenação de Apoio à Pesquisa do Hospital Escola mediante a assinatura do Termo de Concordância da Instituição para participação em Pesquisa e pelo Comitê de Ética em Pesquisa (CEP) através do sistema Plataforma Brasil em 25 de novembro de 2018 por meio do parecer № 3.037.338.

A coleta de dados teve início em dezembro de 2018. Antes da aplicação dos questionários realizou-se préteste com os profissionais do setor de pediatria do referido serviço. Este setor foi previamente escolhido, devido a não participação deste estudo, uma vez que em crianças alguns cuidados preconizadas na literatura sofrem variação como por exemplo o tempo de permanência do CVP. Neste setor de acordo com a CCIH e o Plano Operacional Padrão (POP) do serviço, se o cateter periférico estiver identificado, limpo e sem sinais flogísticos, o tempo de permanência poderá ser estendido para mais de 96 horas.

Procedeu-se a leitura e coleta da assinatura dos sujeitos no Termo de Consentimento Livre e Esclarecido para participação em pesquisa. Os dados foram coletados em momentos de maior conveniência para os sujeitos e pesquisadores, de forma a não atrapalhar as atividades dos profissionais.

A análise estatística descritiva de frequência dos dados foi processada pelo software SPSS (Statistical Package for the Social Sciences) para Windows, versão 20.0, considerando-se um nível de significância de $5 \%(a=0,05)$. Foi realizada análise com teste Qui-quadrado de Pearson $\left(X^{2}\right)$ para comparar as características sociodemográficas e funcionais com o conhecimento dos profissionais.

\section{RESULTADOS}

O pré-teste foi realizado objetivando-se identificar coerência entre as questões contidas nos questionários e se estas atendiam ao objetivo proposto por este estudo. Após a aplicação do mesmo, os questionários foram lidos integralmente pelos pesquisadores e percebeu-se a necessidade de alteração apenas no layout dos instrumentos.

Participaram deste estudo 189 profissionais de Enfermagem, sendo a maioria do sexo feminino 120 $(63,5 \%)$. Sobre a faixa etária, estes apresentaram idades entre 28 e 62 anos, a maioria entre 40 a 62 anos, 111 (58\%), com predominância de 37 a 48 anos.

Quanto à profissão, $31(16,4 \%)$ eram enfermeiros e 158 (83,6\%) técnicos em Enfermagem. O maior número de profissionais $166(87,8 \%)$ trabalhava no hospital há mais de 7 anos. Sobre o turno de trabalho 108 $(57,1 \%)$ trabalhavam no plantão diurno e 86 (54,5\%) não possuíam outro vínculo empregatício.

Em relação ao tempo de trabalho, prevaleceu os que trabalhavam há mais que 7 anos na instituição 166 (87,8\%). Os setores que apresentaram maior número de colaboradores foram PS 49 (31,6\%), seguido da CM $40(25,8 \%)$ e UTI 31 (20\%). O número de pacientes para cada funcionário variou de acordo com cada setor, mas a maioria dos profissionais $65(34,4 \%)$ referiu cuidar de 4 a 6 pacientes por plantão, mas vale lembrar que na UTI cada técnico fica responsável por somente 2 pacientes. Também foi perguntado sobre o vínculo empregatício, na época do estudo 103 (54,5\%) trabalhavam somente no local da pesquisa.

Quando questionados sobre identificação do acesso venoso 179 (94,7\%) dos profissionais afirmaram que sempre identificavam o curativo do AVP. $121(64,0 \%)$ descreveram que o tempo ideal de permanência do cateter é de 96 horas. Em relação ao material mais adequado para fixação do cateter, a maioria, 138 (73,0 \%) relatou que é o esparadrapo e/ou fita microporosa e apenas $51(27,0 \%)$ responderam fita transparente semipermeável estéril. 
Referindo-se à flebite, 188 (99,5\%) dos profissionais relataram saber identificá-la, mas ao questioná-los sobre os sinais/sintomas descritos na literatura e que são utilizados para identificação desta complicação apenas $94(49,7 \%)$ referiram os sinais/sintomas corretos.

Sobre as lesões cutâneas pós infiltrativas, infiltração e extravasamento $185(97,9 \%)$ afirmaram saber identificar a sua ocorrência, mas, apenas $82(43,4 \%)$ demarcaram corretamente os sinais e sintomas dessas complicações.

Sobre a variável "Tempo em que trabalha no hospital", 15 (16,0\%) dos profissionais com menos de 7 anos na instituição conheciam os sinais e sintomas que caracterizam corretamente a flebite e somente $8(8,4 \%)$ demarcaram a alternativa errada. No entanto, deve ser levado em consideração que estes eram também o menor número de profissionais, porém, tiveram resultado mais satisfatório comparando com os que trabalhavam por um período de tempo maior no serviço (Tabela 1).

A variável profissão apresentou-se estatisticamente significativa em relação ao conhecimento correto dos sinais e sintomas de flebite ( $p: 0,003)$. Já, para infiltração/extravasamento o resultado foi extremamente significante ( $p: 0,000)$. Ou seja, os enfermeiros conhecem mais sobre esses eventos adversos. Na variável "Possui outro vínculo empregatício" tanto aqueles que trabalham somente no serviço pesquisado quanto os que tinham outro vínculo empregatício responderam erroneamente sobre extravasamento e infiltração (Tabela 1).

Outra análise de dependência realizada foi do conhecimento sobre o material ideal para fixação dos cateteres e a categoria profissional, nesse quesito, 24 (47,1\%) dos enfermeiros acertaram e somente $7(5,1 \%)$ erraram. Se comparando com os técnicos em enfermagem, novamente a profissão foi extremamente significante (p: 0,000) (Tabela 2).

Sobre a análise de associação entre o tempo de permanência do cateter e as variáveis independentes (idade, profissão, tempo que trabalha no hospital, número de pacientes por plantão e o vínculo empregatício) nenhum resultado foi estatisticamente significativo, ou seja, existe um bom conhecimento sobre o tempo ideal de permanência do cateter diante do maior número de acertos (Tabela 2). 
Tabela 1 - Análise de associações entre os sinais utilizados corretamente e erroneamente para identificação de Flebite e Infiltração/ Extravasamento pelos profissionais da enfermagem de um Hospital Escola de Montes Claros- MG, Brasil. 2019, n= 189.

\begin{tabular}{|c|c|c|c|c|c|c|c|c|}
\hline \multirow[b]{2}{*}{ Variáveis independentes } & \multicolumn{4}{|c|}{ Sinais de Flebite } & \multicolumn{4}{|c|}{ Sinais Infiltração/Extravasamento* } \\
\hline & $\begin{array}{c}\text { Correto } \mathrm{n} \\
(\%)\end{array}$ & $\begin{array}{c}\text { Errado } \\
\mathrm{n}(\%)\end{array}$ & $\begin{array}{l}\text { Total } \\
\mathrm{n}(\%)\end{array}$ & $\mathbf{p}^{*}$ & $\begin{array}{c}\text { Correto } \\
\mathrm{n}(\%)\end{array}$ & $\begin{array}{c}\text { Errado } \\
\mathrm{n}(\%)\end{array}$ & $\begin{array}{l}\text { Total } \\
\mathrm{n}(\%)\end{array}$ & $\mathbf{p}^{*}$ \\
\hline \multicolumn{9}{|l|}{ Idade (anos)* } \\
\hline De anos 28 a 39 & $39(43,8)$ & $26(29,9)$ & $65(39,9)$ & \multirow{3}{*}{,055 } & $32(43,2)$ & $33(32,7)$ & $65(37,1)$ & \multirow{3}{*}{, 153} \\
\hline De 40 a 62 & $50(56,2)$ & $61(70,1)$ & $111(63,1)$ & & $42(56,8)$ & $68(67,3)$ & $110(62,9)$ & \\
\hline Total & $89(100)$ & $87(100)$ & $176(100)$ & & $74(100)$ & $101(100)$ & $175(100)$ & \\
\hline \multicolumn{9}{|l|}{ Profissão } \\
\hline Enfermeiro & $23(24,5)$ & $8(8,4)$ & $31(16,4)$ & \multirow{3}{*}{,003 } & $25(30,5)$ & $6(5,7)$ & $31(16,5)$ & ,0 \\
\hline Técnico em Enfermagem & $71(75,5)$ & $87(91,6)$ & $158(83,6)$ & & $57(69,5)$ & $100(94,3)$ & $157(83,5)$ & \multirow{2}{*}{$\begin{array}{l}0 \\
0\end{array}$} \\
\hline Total & $94(100)$ & $95(100)$ & $189(100)$ & & $82(100)$ & $106(100)$ & $188(100)$ & \\
\hline \multicolumn{9}{|l|}{$\begin{array}{c}\text { Tempo em que trabalha no } \\
\text { hospital }\end{array}$} \\
\hline Menos que 7 anos & $15(16,0)$ & $8(8,4)$ & $23(12,2)$ & \multirow{3}{*}{, 113} & $6(7,3)$ & $17(16,0)$ & $23(12,2)$ & \multirow{3}{*}{,070 } \\
\hline Mais que 7 anos & $79(84,0)$ & $87(91,6)$ & $166(87,8)$ & & $76(92,7)$ & $89(84,0)$ & $165(87,8)$ & \\
\hline Total & $94(100)$ & $95(100)$ & $189(100)$ & & $82(100)$ & $106(100)$ & $188(100)$ & \\
\hline \multicolumn{9}{|l|}{$\begin{array}{l}\text { Número de pacientes por } \\
\text { plantão sob sua } \\
\text { responsabilidade }\end{array}$} \\
\hline Menos que 7 & $48(51,1)$ & $46(48,4)$ & $94(49,7)$ & \multirow{3}{*}{,716 } & $48(58,5)$ & $46(43,4)$ & $94(50,0)$ & \multirow{2}{*}{, 03} \\
\hline Mais que 7 & $46(48,9)$ & $49(51,6)$ & $95(50,3)$ & & $34(41,5)$ & $60(56,6)$ & $94(500)$ & \\
\hline Total & $94(100)$ & $95(100)$ & $189(100)$ & & $82(100)$ & $106(100)$ & $188(100)$ & 9 \\
\hline \multicolumn{9}{|l|}{$\begin{array}{c}\text { Possui outro vínculo } \\
\text { empregatício }\end{array}$} \\
\hline Sim & $43(45,5)$ & $43(45,3)$ & $86(45,5)$ & \multirow{3}{*}{, 947} & $36(43,9)$ & $50(47,2)$ & $86(45,7)$ & \multirow{3}{*}{,656 } \\
\hline Não & $51(54,3)$ & $52(54,7)$ & $103(54,5)$ & & $46(56,1)$ & $56(52,8)$ & $102(54,3)$ & \\
\hline Total & $94(100)$ & $95(100)$ & $189(100)$ & & $82(100)$ & $106(100)$ & $188(100)$ & \\
\hline
\end{tabular}

Legenda: ${ }^{*}$ Missing System ${ }^{*}$ : Qui-quadrado de Pearson (Valor de significância).

Fonte: Gomes BKG, et al., 2019. 
Tabela 2 - Análise de associações entre o material ideal usado para fixação do cateter e tempo de permanência do cateter pelos profissionais da enfermagem de um Hospital Escola de Montes Claros- MG, Brasil. $2019(n=189)$.

\begin{tabular}{|c|c|c|c|c|c|c|c|c|}
\hline \multirow[b]{2}{*}{ Variáveis independentes } & \multicolumn{2}{|c|}{ Material de fixação ideal } & \multicolumn{6}{|c|}{ Tempo de permanência do cateter } \\
\hline & $\begin{array}{c}\text { Correto } n \\
(\%)\end{array}$ & $\begin{array}{c}\text { Errado } \\
\mathrm{n}(\%)\end{array}$ & $\begin{array}{l}\text { Total } \\
\mathrm{n}(\%)\end{array}$ & $\mathbf{p}^{*}$ & $\begin{array}{c}\text { Correto } \\
\mathrm{n}(\%)\end{array}$ & $\begin{array}{c}\text { Errado } \\
\mathrm{n}(\%)\end{array}$ & $\begin{array}{l}\text { Total } \\
\mathrm{n}(\%)\end{array}$ & $\mathbf{p}^{\star}$ \\
\hline \multicolumn{9}{|l|}{ Idade (anos) ${ }^{\star}$} \\
\hline De anos 28 a 39 & $22(46,8)$ & $43(33,3)$ & $65(36,9)$ & \multirow{3}{*}{, 101} & $45(39,1)$ & $20(32,8)$ & $65(36,9)$ & \multirow{3}{*}{,407 } \\
\hline De 40 a 62 & $25(53,2)$ & $86(66,7)$ & $111(63,1)$ & & $70(60,9)$ & $41(67,2)$ & $111(63,1)$ & \\
\hline Total & $47(100)$ & $129(100)$ & $176(100)$ & & $115(100)$ & $61(100)$ & $176(100)$ & \\
\hline \multicolumn{9}{|l|}{ Profissão } \\
\hline Enfermeiro & $24(47,1)$ & $7(5,1)$ & $31(16,4)$ & \multirow{3}{*}{, 000} & $23(19)$ & $8(11,8)$ & $31(16,4)$ & \multirow{3}{*}{197} \\
\hline Técnico em Enfermagem & $27(52,9)$ & $131(94,9)$ & $158(83,6)$ & & $98(81)$ & $60(88,2)$ & $158(83,6)$ & \\
\hline Total & $51(100)$ & $138(100)$ & $189(100)$ & & $121(100)$ & $68(100)$ & $189(100)$ & \\
\hline \multicolumn{9}{|l|}{$\begin{array}{c}\text { Tempo em que trabalha } \\
\text { no hospital }\end{array}$} \\
\hline Menos que 7 anos & $5(9,8)$ & $18(13)$ & $23(12,2)$ & \multirow{3}{*}{, 545} & $15(12,4)$ & $8(11,8)$ & $23(12,2)$ & \multirow{3}{*}{899} \\
\hline Mais que 7 anos & $46(90,2)$ & $120(87,0)$ & $166(90,2)$ & & $106(87,6)$ & $60(68)$ & $166(87,8)$ & \\
\hline Total & $51(100)$ & $138(100)$ & $189(100)$ & & $121(100)$ & $68(100)$ & $189(100)$ & \\
\hline \multicolumn{9}{|l|}{$\begin{array}{l}\text { Número de pacientes } \\
\text { por plantão sob sua } \\
\text { responsabilidade }\end{array}$} \\
\hline Menos que 7 & $26(51)$ & $68(49,3)$ & $94(49,7)$ & \multirow{3}{*}{ 835 } & $59(48,8)$ & $35(51,5)$ & $94(49,7)$ & \multirow{3}{*}{,721 } \\
\hline Mais que 7 & $25(49)$ & $70(50,7)$ & $95(50,3)$ & & $62(51,2)$ & $33(48,5)$ & $95(50,3)$ & \\
\hline Total & $51(100)$ & $138(100)$ & $189(100)$ & & $121(100)$ & $68(100)$ & $189(100)$ & \\
\hline \multicolumn{9}{|l|}{$\begin{array}{l}\text { Possui outro vínculo } \\
\text { empregatício }\end{array}$} \\
\hline Sim & $21(41,2)$ & $65(47,1)$ & $86(45,5)$ & \multirow{3}{*}{,788 } & $58(47,9)$ & $28(41,2)$ & $86(45,5)$ & \multirow{3}{*}{,371 } \\
\hline Não & $30(58,8)$ & $73(52,9)$ & $103(54,5)$ & & $63(52,1)$ & $40(58,8)$ & $103(54,5)$ & \\
\hline Total & $51(100)$ & 138(100) & $189(100)$ & & $121(100)$ & $68(100)$ & $189(100)$ & \\
\hline
\end{tabular}

Legenda: *Missing System $\mathrm{p}^{*}$ Qui-quadrado de Pearson (Valor de significância).

Fonte: Gomes BKG, et al., 2019. 


\section{DISCUSSÃO}

Foram encontrados poucos estudos nacionais atuais publicados sobre o conhecimento da equipe de enfermagem sobre acesso venoso periférico. Em relação ao método, nos estudos encontrados, houve predomínio da abordagem exploratória, randomizada, estudo de coorte, observacional ou revisão sistemática. Estudo de Melo EM, et al (2015), realizado com profissionais de enfermagem em um Hospital público de Fortaleza - Ceará que objetivou analisar os cuidados realizados pelos profissionais de enfermagem durante a punção de acesso venoso periférico, apresentou dados semelhantes aos encontrados neste estudo, onde a maioria de sujeitos eram do sexo feminino $83(90,2 \%)$, com idades entre 41 a 50 anos.

Sobre a categoria profissional, o mesmo estudo, demonstrou uma maioria de técnicos em enfermagem, o que compreendeu mais da metade da amostra 158 (83,6\%). O maior número dos profissionais de enfermagem de nível técnico está associado ao grau de suas funções, frequentemente de menor complexidade, porém com maior demanda. O enfermeiro desempenha o papel de coordenador da equipe de enfermagem, realizando atividades de maior exigência técnico-científica (PEREIRA KC, et al., 2016).

Neste estudo a maioria dos profissionais relataram identificar o AVP, prática fundamental para que se mantenha em condições ideais e permita que a equipe de saúde tenha acesso aos dados referentes ao dispositivo, contribuindo para que se evite o desenvolvimento de complicações (PEREIRA KC, et al., 2016). Em contrapartida, estudo de Rós ACR, et al (2017), avaliou os cuidados de enfermagem na terapia intravenosa periférica em idosos em um hospital escola do sul do Brasil, e o resultado foi a baixa conformidade quanto identificação, apenas 15 (18,8\%) estavam adequados.

Em 2010, o Conselho Regional de Enfermagem de São Paulo promoveu uma campanha em prol da segurança do paciente e uma das medidas estabelecidas foi a identificação do AVP contendo registro da data de punção, o número do calibre do cateter usado e a identificação do profissional que realizou o procedimento (OLIVEIRA ECS, et al., 2016).

O tempo ideal de permanência do CVP foi considerado adequado como de 96 horas para $121(64,0 \%)$ dos participantes. A ANVISA, estabelece que esse é o tempo máximo para a troca do mesmo, sendo que, a decisão pelo aumento deste tempo deverá ser realizada somente quando houver indicação clínica da Comissão de Controle de Infecção Hospitalar de cada instituição (AGÊNCIA NACIONAL DE VIGILÂNCIA SANITÁRIA, 2013). Por outro lado, alguns estudos afirmam que o tempo limite para troca é de $72 \mathrm{~h}$, prazo em que o cateter ainda se mantém pérvio, se a manutenção estiver apropriada, ou então houver dificuldade para se conseguir outro acesso este prazo poderá ser estendido para até 96 horas, desde que, esteja em perfeitas condições (OLIVEIRA ECS, et al., 2016; RÓS ACR, et al., 2017).

Além disto, em uma revisão sistemática, em 7 dos 19 estudos realizados por Oliveira ASS e Parreira PMSD (2010), foi encontrada uma associação estatisticamente significativa entre flebites e o maior tempo de permanência do cateter, dessa forma, o tempo de permanência deste dispositivo não deve ser negligenciado pela equipe de enfermagem.

O material mais adequado para fixação do cateter, de acordo com $138(73,0 \%)$ dos sujeitos foi o esparadrapo e/ou fita microporosa, este não é o material recomendado, mas, de acordo com POP e normas institucionais do hospital cenário do estudo, a fita transparente semipermeável estéril não é preconizada para fixação do CVP.

A cobertura para cateter periférico deve ser estéril, podendo ser semi-oclusiva (gaze e fita adesiva estéril) ou uma membrana transparente semipermeável (AGÊNCIA NACIONAL DE VIGILÂNCIA SANITÁRIA, 2013; RÓS ACR, et al., 2017). A utilização desses curativos possibilita uma melhor visualização do local de inserção do cateter o que garante avaliação constante pela enfermagem e a detecção precoce de sinais e sintomas que alertam para a possibilidade de inflamação ou infecção (OLIVEIRA ECS, et al., 2016).

Observou-se que quase não são utilizadas coberturas estéreis para a fixação do CVP nas instituições de saúde do Brasil mesmo sendo uma recomendação da ANVISA (RÓS ACR, et al., 2017). Habitualmente são usados esparadrapo e fita microporosa para manter o cateter fixado por mais tempo devido a dificuldade de manutenção e aquisição dos curativos estéreis de poliuretano transparente (SOUSA N, et al., 2019). Os 
enfermeiros se sobressaíram quanto aos conhecimentos corretos sobre flebite, extravasamento e infiltração. Isso pode estar relacionado ao maior aprofundamento do conhecimento teórico realizados nas academias, o que favorece uma maior qualidade na assistência.

As questões teórico/científicas são reconhecidas como próprias do enfermeiro e garantem maior identidade a este profissional. A ampliação dos conhecimentos através da leitura de publicações científicas garante a este profissional um papel de destaque dentro da equipe de enfermagem e faz com que este contribua para que o conhecimento produzido chegue à realidade dos serviços de saúde, da comunidade e dos centros de formação (SOUSA N, et al., 2019). Cabe ao enfermeiro avaliar criteriosamente os riscos de flebite, infiltração e extravasamento em seus pacientes, assim como, capacitar toda a equipe além de propor os cuidados necessários quando da sua ocorrência. Por isso, faz-se necessário que o enfermeiro alie conhecimentos teóricos a habilidades técnicas (TERTULIANO AC, et al., 2014).

Em uma pesquisa prospectiva realizada por Tertuliano AC (2014), em um hospital do Vale do Paraíba, a porcentagem de flebite foi de $31,6 \%$, considerada elevada quando comparada ao padrão aceito pela Intravenous Nurses Society que é de $5 \%$. Pesquisa realizada em um hospital escola do sul do Brasil a ocorrência de flebite e extravasamento foi de 7,5\% (RÓs ACR, et al., 2017). No estudo de Braga LM, et al (2018), sobre o indicador de qualidade sensível aos cuidados de Enfermagem foi identificado a incidência para flebite de $11,5 \%$ e infiltração $15,8 \%$ numa amostra de 110 sujeitos. Uma limitação foi o desenvolvimento de estudo do tipo transversal, contudo, salienta-se que o objetivo proposto foi atingido.

\section{CONCLUSÃO}

Neste estudo observou-se que o conhecimento teórico sobre a necessidade de identificação do AVP e do tempo adequado de permanência do mesmo encontra-se solidificado entre os profissionais de enfermagem. Observou-se ainda, que existe uma lacuna quanto ao conhecimento apresentado pelos profissionais sobre 0 tipo de curativo ideal para fixação do cateter, os sinais e sintomas característicos de flebite, infiltração e extravasamento. Os serviços de saúde devem proporcionar capacitação permanente principalmente para os técnicos em enfermagem e estes devem ter como foco a prestação de cuidados de enfermagem de forma a garantir a segurança dos pacientes.

Sugere-se que, seja realizado, neste mesmo serviço, um estudo observacional com o objetivo de verificar se 0 conhecimento apresentado pelos profissionais de enfermagem em relação à adequada manutenção do AVP é realmente aplicado durante sua prática assistencial. Sugere-se ainda, que o serviço estudado padronize o curativo filme transparente não estéril que por ser transparente facilita a visualização e monitorização do local de inserção do cateter, resultando em deteç̧ão precoce dos sinais/sintomas de complicações e consequentemente de intervenção adequada para resolução deste problema.

\section{REFERÊNCIAS}

1. AGÊNCIA NACIONAL DE VIGILÂNCIA SANITÁRIA. Medidas de Prevenção de Infecção Relacionada à Assistência à Saúde. Caderno 4. Brasília: Anvisa, 1ed; 2013; 92p.

2. AGÊNCIA NACIONAL DE VIGILÂNCIA SANITÁRIA. Assistência Segura: Uma Reflexão Teórica Aplicada a Pratica. Brasília: Anvisa, 2ed; 2017; 168p.

3. BARBOSA M, et al. Quality indicators in support of intravenous therapy in a university hospital: a contribution of nursing. Rev de Pesquisa: Cuidado é Fundamental Online, 2015; 7(2): 2277-2286.

4. BARBOSA MH, et al. Clima de segurança do paciente em unidades de clínica médica e cirúrgica. Revista Eletrônica De Enfermagem REE, 18. 2016.

5. BITENCOURT ES, et al. Prevalência de flebite relacionada ao uso de dispositivos intravenosos periféricos em crianças. Cogitare enferm, 2018; 23(1): 1-9.

6. BRAGA LM, et al. Flebite e infiltração: traumas vasculares associados ao cateter venoso periférico. Rev. Latino-Am. Enferm. 2018; 26: e3002.

7. DANSKI MTR, et al. Complicações relacionadas ao uso do cateter venoso periférico: ensaio clínico randomizado. Acta Paul. Enferm. 2016a; 29(1): 84-92.

8. DANSKI MTR, et al. Incidência de complicações locais e fatores de risco associados ao cateter intravenoso periférico em neonatos. Rev. Esc. Enferm. USP, 2016b; 50(1): 22-28. 
9. ENES SMS, et al. Flebite associada a cateteres intravenosos periféricos em adultos internados em hospital da Amazônia Ocidental Brasileira. Rev. Esc. Enferm. USP, 2016; 50(2): 263-271.

10. FEREZIN TPM, et al. Análise da notificação de eventos adversos em hospitais acreditados. Rev. Cogitare Enferm. $2017 ; 2(22): 49644$.

11. INFUSION NURSES SOCIETY. Infusion Therapy Standards of Practice. Journal of Infusion Nursing. Sandy, Utah: 2016; 39(1S): S1- S59. Supplement to ISSN 1533-1458. 169p.

12. MELO EM, et al. Cuidados dispensados pela equipe de enfermagem durante o procedimento de punção venosa periférica. Rev. Enferm. UFPE, 2015; 9(3):1022-30.

13. MURASSAKI ACY, et al. Avaliação de cuidados na terapia intravenosa: desafio para a qualidade na enfermagem. Esc. Anna Nery. 2013; 17(1): 11-16.

14. OLIVEIRA ASS, PARREIRA PMSD. Intervenções de enfermagem e flebites decorrentes de cateteres venosos periféricos. Revisão sistemática da literatura. Rev. Enf. 2010; serllI(2): 137-147.

15. OLIVEIRA ECS, et al. Caracterização das flebites notificadas à gerência de risco em hospital da rede Sentinela. Rev. Baiana de Enfer. 2016; v(3):15361: 1-9.

16. PEREIRA KC, et al. Análise crítica dos vídeos sobre punção venosa periférica com cateter disponibilizados no Youtube. REME - Rev Min Enferm. 2016; 20:e970.

17. RÓS ACR, et al. Terapia intravenosa m idosos hospitalizados: Avaliação de cuidados. Rev. Cogitare Enferm. 2017; 2(22): 49989.

18. SANTOS DS, et al. Segurança do Paciente Idoso Hospitalizado: Uma Revisão Integrativa. Cogitare Enferm. 2016; 21(3): 01-10.

19. SILVA ACA, et al. A segurança do paciente em âmbito hospitalar: revisão integrativa da literatura. Rev. Cogitare Enferm. 2016; 21(5): 01-09.

20. SOUSA N, et al. Enfermagem e ciência: uma reflexão sobre sua consolidação. Rev. de Enfer da UFPE on-line. 2019; $13(3)$ : 839-843.

21. TERTULIANO AC, et al. Flebite em acessos venosos periféricos de pacientes de um hospital do Vale do Paraíba. Rev. Min Enferm. 2014; 18(2): 334-339.

22. VITURI DW, MATSUDA LM. Validação de conteúdo de indicadores de qualidade para avaliação da assistência de enfermagem. Rev. Esc. Enferm. USP. 2009; 43(2): 429-437. 